\title{
Microaneurysms Detection using Blob Analysis for Diabetic Retinopathy
}

\author{
Saripah Abu Bakar', Haniza Yazid ${ }^{2, *}$, Saufiah Abd Rahim ${ }^{3},{ }^{4}$ Shafriza Nisha \\ Basah
}

1,2,3,4 School of Mechactronic Engineering, Universiti Malaysia Perlis, Campus Ulu Pauh, 02600 Arau Perlis, MALAYSIA.

*Corresponding author

DOI: https://doi.org/10.30880/ijie.2019.11.06.021

Received 16 July 2018; Accepted 21 July 2019; Available online 12 September 2019

\begin{abstract}
Blob analysis is a mathematical method to find the region of interest (ROI) by focusing on the characteristics like brightness or colour. In this work, the process to segment Microaneurysms (MAs) involves two stages, which are pre-processing and segmentation. Pre-processing is a phase for noise removal and illumination correction. In this work, several methods were utilized namely Contrast Limited Adaptive Histogram Equalization (CLAHE), Normalization for contrast enhancement and median filter for noise removal. Then, continue with segmentation phase to segment the MAs from the image. In segmentation phase, several methods were used namely morphological opening, thresholding, Hessian Matrix 2D and Eigenvalue of Hessian Matrix. Finally all the resulting images were compared with the benchmark image to measure the accuracy and grading the stage of Diabetic Retinopathy (DR) by comparing the number of detected MAs. The segmentation accuracy of this project is $68 \%$ and $55 \%$ accuracy for stage grading.
\end{abstract}

Keywords: blob analysis, image segmentation, noise removal, microanerysms

\section{Introduction}

Diabetic Retinopathy (DR) is one of the diabetic's disease. DR is known as diabetic's eye illness which can develop to all diabetic patients. Usually, DR will attack diabetic patient who suffers diabetes mellitus or type two diabetics for a long time. Medical professional divided DR into two subgroups which are Non-Proliferate Diabetic Retinopathy (NPDR) and Proliferate Diabetics Retinopathy (PDR) [1]. The development of Microaneurysms (MAs) is one of the earliest symptom of DR. The early detection of MAs is necessary and vital because if the visual examination able to detect at least one MA inside the retina, it can reveal information about the retinal disease [1]. MAs appear as a small circular dots and the colour of MAs is dark reddish. Based on clinical ophthalmologist, the size of MAs usually is in the range (34 \pm $16 \mu \mathrm{m})$, and $60 \%$ smaller than $40 \mu \mathrm{m}[1,2]$.

In pre-processing phase, several methods were proposed in order enhance the retinal image. According to Wan Azani et. al, their studies focused on illumination correction. They used combination of Gaussian filtering and low pass filter for normalizing the background. The researchers also applied Gaussian filter to remove the noise. The sigma value will affect the brightness of the image. In their work, the sigma value that they used is 0.5 . Based on finding, if the sigma value is high the image will be blurred and darken [3]. Lee, Pant, and Lee [4] proposed a method to enhance the contrast that can be applied in medical image and natural image. The study is the continuous and improvement from previous methods which are Histogram Equalization (HE) and Adaptive Histogram Equalization (AHE). In their work, the parameters that they utilized were size of contextual region and clip limit of histogram CLAHE with local image contrast preserving dynamic range compression. The advantage of this method is the possibility of under-enhancement and over 
enhancement to happen were less. This is because the range of the gain parameter is transformed over the edge density of the image.

Fleming et al. [5] proposed a contrast normalization method in order to detect and distinguish the MAs and other dots inside the retina. Firstly, they compared the contrast surrounding the retina. If the dot has similar intensity with the retina it will not conclude as the MAs. Besides, in order to avoid miss detection with noise, they used Gaussian filtering method with sigma value equal to 0.2 and median filter. Morphological top hat and watershed were chosen to segment the MAs from the retina surrounding. Moreover, for blob detection, they used edge growing method to detect the MAs.

Datta et al. [6] proposed Contrast Limited Adaptive Histogram Equalization (CLAHE) for contrast correction. Firstly the retina image was converted into green channel. From their studies, green channel was chosen to be used for further processing. The blue channel is not suitable because of low contrast and lack of information. Besides, red channel contained a lot of noise and simply saturated. Next, they proposed an algorithm that divide the number of retina fundus image into a small and non-overlapping contextual region. Median filter was used to smooth the image background. Median filter was utilized because the ability to remove the noise and smooth the image without creating a new unrealistic pixel intensity.

Inoue et.al, proposed MAs detection based on Eigenvalue analysis using Hessian matrix and combination of double ring filter but the output images contained many false detection. Rubini et. al, proposed the detection of MAs based on Hesian Matrix, two approaches have been done which are semi-automated Hessian-based candidates selection algorithm followed by thresholding to detect true MAs and Hemorrhages (HR) [7]. Kedir et. al, proposed robust MAs candidates based on blob detection theory which have Hessian matrix and Singular Value Decomposition (SVD) then continue with SVM classifier [8]. Wang et. al proposed a method that differentiate the MAs from the background by applying thresholding on number of pixel using Parzen Window estimation technique [9]. Mazlan et.al proposed an approach that utilizing several techniques such as filtration, background exclusion, contrast enhancement, h-maxima transform, multilevel thresholding and morphological closed operation. The percentage of severity grade that correctly detected when compared to ground truth grading is $60 \%$ [10].

\section{Methodology}

In this study there are 20 retina images with 960 x 1440 pixel were taken from E-OPTHA database. Each image contains difference illumination problem. Based on the benchmark, there are 249 MAs in 20 of the retinal images. All images contained at least one MAs. The main objectives of this paper is to detect MAs by using blob analysis. In this work, the main focuses is the pre-processing and segmentation phase. All programs were developed using MATLAB software.

\subsection{Pre-processing}

\section{(i) Green Channel Conversion}

Images represent in primary colour which are Red, Green and Blue. The primary colour is known as RGB. Among three channels, green channel is the best channel to be used to detect the MAs since it has the best background contrast [2]. Green channel pixels value are extracted using eq. 1:

Where:

$$
\mathrm{g}=G /(R+G+B)
$$

- $g=$ green channel

- $R=\operatorname{Red}$

- $G=$ Green

- $B=$ Blue

\section{(ii) Complement of image}

Complement image is the process of subtracting the maximum pixel inside the image with each pixel inside the particular image. Lighter image becomes darker and the darker will become lighter. Usually, complement image can be applied to the image that have poor contrast.

\section{(iii) Normalization}

Normalization is a technique to enhance the contrast due to poor illumination. The normalization technique work by changing and stretching the intensity value then make it full of possible value [11]. The normalization work by using the following equation:

Normalization $=($ I-Min $) \frac{\text { new Max-new Min }}{\text { Max-Min }}+$ new min 
Where:

- $\quad I=$ Normalization in grey scale image

- $\quad$ new Min = new minimum intensity in range

- $n e w$ Max = new maximum intensity in range

- $M a x=$ maximum intensity in range

- $\quad M i n=$ minimum intensity in range

\section{(iv) CLAHE}

CLAHE is the method to improve the visualization quality [12]. The process works by dividing the sub image into several sub images. Then, the histogram is used to compute the difference between the boundary sections of the image. Next, the clip limit of the histogram is set at predefine value in order to limit the amplification.

\section{(v) Median filter}

The unwanted noise may developed during the acquisition process. In this work, the median filter was utilized. Median filter was implemented by sorting all the pixels value in increasing order then replace with the middle value of the pixel $[12,13]$.

\section{(vi) Morphological operation}

Since inside the retina consists of blood vessels and optical disk, both optical disk and blood vessels need to be eliminated in order to make the detection process of MAs become easier. Morphological opening involves combination of erosion followed by dilation process. Thus, all the weak line/element will be removed then, growth back the remaining elements inside the image. Structuring element plays an important role because the successful of this method depends on the structuring element [14]. Firstly, for removing optical disk, the structuring element that suitable is disk with 8 pixel as the diameter [14]. Since the optical disk have round shape. After that, for removing blood vessel the connecting pixels that is smaller than 5 pixel will be enhanced first, then the image from thresholding will subtract that connecting pixel.

\subsection{Segmentation - Thresholding}

Thresholding method is a process to differentiate the image or object from their background. Usually, the output from the image will be in binary form $[0,1]$ which is black or white. Thresholding operates by segment the grey level of the image into two sub-region [13]. The mathematical formula for global thresholding is given by the following equation:

$g(x, y)\left\{\begin{array}{ll}1 & \text { if } f(x, y)>t h \\ 0 & \text { if } f(x, y) \leq t h\end{array}\right\}$

Where:

- $g(x, y)=$ Output image.

- $f(x, y)=$ input image.

- $\quad t h=$ Thresholding values.

\subsection{Post-preprocessing}

Post pre-processing was utilized since there are remaining blood vessel and noise after the segmentation. Therefore, median filter was used once again to remove unnecessary artifacts.

\section{(i) Hessian matrix 2D}

After complete the pre-processing stage, Hessian matrix was used in order to detect the MAs. Hessian matrix is a second order partial derivative. In order to detect the MAs Hessian matrix was applied first before continue with eigenvalue of 
Hessian Matrix. The Hessian Matrix method not only detected blob like structure, but it also detected line like (vessel) and plate like structure [7, 15]. The formula for Hessian matrix is given by:

$H(x, y)=\left[\begin{array}{ll}L(x, y) & L(x, y) \\ L(x, y) & L(x, y)\end{array}\right]$

$L_{x x}=G_{x x}(x, y) * I(x, y)$.

$L_{x y}=G_{x y}(x, y) * I(x, y)$.

$L_{y x}=G_{y x}(x, y) * I(x, y)$.

Where :

- $G_{x x}(x, y), G_{x y}(x, y), G_{y x}(x, y)=$ second order partial derivative

- $I(x, y)=$ processed image

\section{(ii) Eigenvalue of Hessian Matrix}

The eigenvalue of Hessian matrix will detect the MAs by comparing the characteristics represented by $\lambda 1$ and $\lambda 2$. Then, two eigenvalues of Hessian matrix will classify the shape of an intensity of the surface [2,]. The formula is given by:

$R=\left\{\begin{array}{lr}\frac{-2}{\pi} \tan ^{\wedge}-1\left(\frac{\lambda 1+\lambda 2}{\lambda 1-\lambda 2}\right) \text { if } \lambda 1 \neq \lambda 2 \\ -1 & \text { if } \lambda 1=\lambda 2>0 \\ 1 & \text { if } \lambda 1=\lambda 2<0\end{array}\right\}$

Where :

- $\quad R=$ Shape intensity.

- $\lambda 1, \lambda 2=$ Eigenvalue of Hessian matrix.

Based on the Hessian matrix as the input of the operator to extract the MAs using the mathematical formula as denoted in Table 1. Table 1 shows the relationship between $\lambda 1$ and $\lambda 2$ [12].

Table 1 -Eigenvalue of the hessian matrix and image structure orientation

\begin{tabular}{|c|c|c|}
\hline$(\lambda 1)$ & $(\lambda 2)$ & Structure of Orientation \\
\hline Low (L) & Low (L) & Noise \\
\hline Low (L) & Low (L) & $\begin{array}{c}\text { Bright sheet like } \\
\text { structure }\end{array}$ \\
\hline Low (L) & Low (L) & Dark sheet like structure \\
\hline Low (L) & $\begin{array}{c}\text { High negative } \\
(\mathrm{H}-)\end{array}$ & $\begin{array}{c}\text { Bright tubular like } \\
\text { structure }\end{array}$ \\
\hline $\begin{array}{c}\text { Low (L) } \\
\text { High }\end{array}$ & $\begin{array}{c}\text { High positive } \\
(\mathrm{H}+)\end{array}$ & $\begin{array}{c}\text { Dark tubular like } \\
\text { structure }\end{array}$ \\
negative & $(\mathrm{H}-)$ & $\begin{array}{c}\text { Bright blob like- } \\
\text { structure }\end{array}$ \\
\hline $\begin{array}{c}\text { High } \\
\text { positive } \\
(\mathrm{H}+)\end{array}$ & $\begin{array}{c}\text { High positive } \\
(\mathrm{H}+)\end{array}$ & $\begin{array}{c}\text { Dark blob like structure } \\
\end{array}$ \\
\hline
\end{tabular}

\section{Results and Discussion}

The proposed methods were evaluated in term of segmentation accuracy. Segmentation accuracy is measured based on the number of MAs that have the exact location as benchmark. The accuracy is calculated by using the formula below.

Accuracy $=(\mathrm{TP}+\mathrm{TN}) /(\mathrm{TP}+\mathrm{TN}+\mathrm{FP}+\mathrm{FN})$ 
Where :

$\mathrm{TP}=$ True Positive

$\mathrm{TN}=$ True Negative

FP $=$ False Positive

$\mathrm{FN}=$ False Negative

Figure 1 shows the original image. Meanwhile Figure 2 shows the resulting image after the optic disc removal.

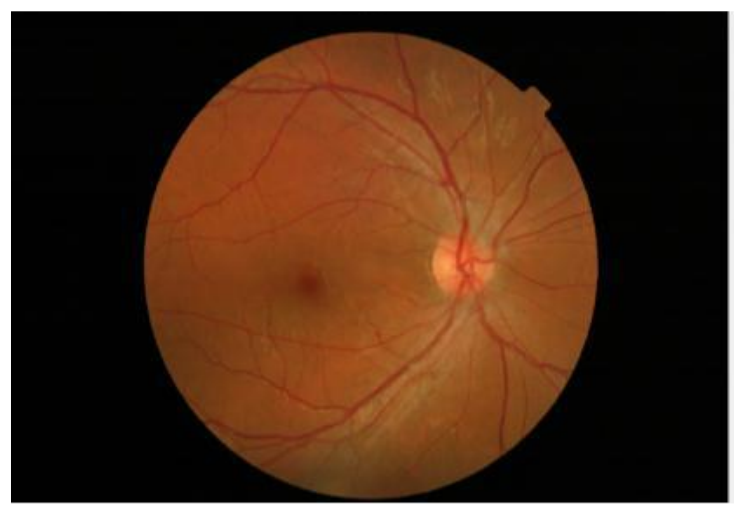

Fig. 1- Original Image



Fig. 2 - Removing Optical disk

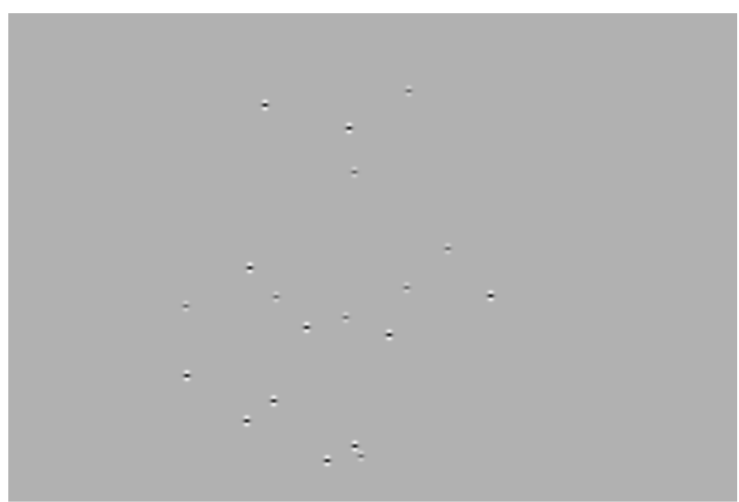

Fig. 3 - Hessian matrix 2D 


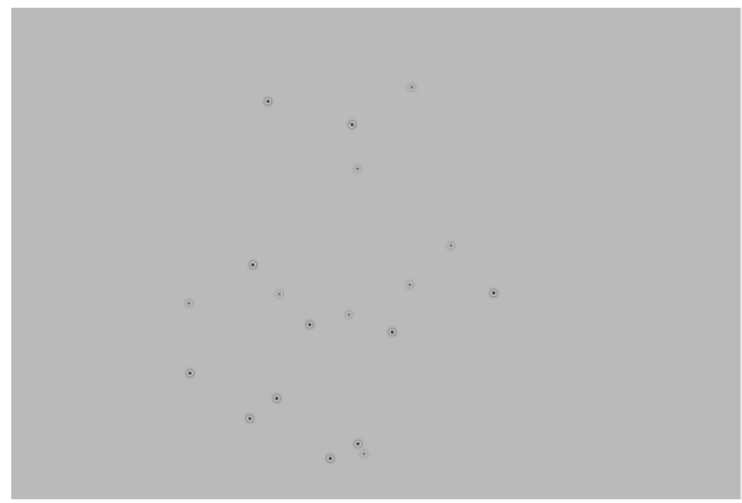

Figure 4 - Eigenvalue of Hessian

Based on the proposed method the accuracy obtained is $69 \%$ when compared the resulting image with benchmark image.

Then, the stage of DR was compared between the segmented MAs and benchmark. The determination of DR stage is referred in Table 2 and overall result is tabulated in Table 3:

Table 2 - Criteria for grading MAs

\begin{tabular}{cll}
\hline Grade & DR Stage & No. MAs \\
\hline 1 & Non. DR & MA $=0$ \\
2 & Mild & $1 \leq \mathrm{MAs} \leq 5$ \\
3 & Moderate & $5<\mathrm{MAs} \leq 15$ \\
4 & Severe & MAs $>15$ \\
\hline
\end{tabular}


Table 3 - Comparison between number of MAs and DR stage

\begin{tabular}{|c|c|c|c|c|}
\hline Image & $\begin{array}{l}\text { Number } \\
\text { of MAs } \\
\text { (segment } \\
\text { ed) }\end{array}$ & $\begin{array}{c}\text { DR stage } \\
\text { (segmente } \\
\text { d) }\end{array}$ & $\begin{array}{c}\text { Number of } \\
\text { MAs } \\
\text { (benchmark } \\
\text { ) }\end{array}$ & $\begin{array}{c}\text { DR stage } \\
\text { (Benchmark) }\end{array}$ \\
\hline 1 & 5 & Moderate & Moderate & 11 \\
\hline 2 & 4 & Mild & Moderate & 6 \\
\hline 3 & 5 & Moderate & Moderate & 7 \\
\hline 4 & 3 & Mild & Moderate & 8 \\
\hline 5 & 25 & Severe & Severe & 34 \\
\hline 6 & 1 & Mild & Mild & 4 \\
\hline 7 & 6 & Moderate & Moderate & 13 \\
\hline 8 & 4 & Mild & Moderate & 9 \\
\hline 9 & 5 & Moderate & Moderate & 10 \\
\hline 10 & 1 & Mild & Mild & 3 \\
\hline 11 & 5 & Moderate & Moderate & 6 \\
\hline 12 & 1 & Mild & Mild & 4 \\
\hline 13 & 14 & Moderate & Severe & 25 \\
\hline 14 & 6 & Moderate & Severe & 16 \\
\hline 15 & 1 & Mild & Mild & 1 \\
\hline 16 & 4 & Mild & Moderate & 10 \\
\hline 17 & 4 & Mild & Moderate & 11 \\
\hline 18 & 3 & Mild & Moderate & 12 \\
\hline 19 & 20 & Severe & Severe & 36 \\
\hline 20 & 14 & Moderate & Severe & 22 \\
\hline
\end{tabular}

From Table 3, it shows the comparison of DR grading between output result and benchmark. There are 129 MAs are correctly identified with exact location as benchmark with 55\% accuracy. By comparing the number of MAs and the DR stage, mostly every candidates images detected less MAs compared to the benchmark due to some MAs were removed during filtering optical disk elimination and blood vessels removal [2, 8]. Therefore, future work can be proposed to improve the enhancement phase in order to reduce the elimination problem. In addition, the original images appear in different contrast. Several images appear as dark image while several images appear as normal. Therefore, the selection of several parameters such as threshold value will affect the process.

\section{Summary}

In conclusion there are many false positive detection of MAs inside the retina image due to some of true positive of MAs was removed during some processes in segmentation and false detected background as MAs. Besides, Hessian matrix 2D and the Eigenvalue of Hessian matrix are the blob analysis method. Since the evaluation result are moderate, it can be conclude that, a good enhancement methods are needed since the images appear in different contrast and variation.

\section{Acknowledgement}

The author would like to acknowledge the support from the Fundamental Research Grant Scheme (FRGS) under a grant number of FRGS/1/2015/SKK06/UNIMAP/02/2 from the Ministry of Higher Education Malaysia. 


\section{References}

[1] F. I. Mustapa, S. Azmi, N. J. N. Mahr. A. Goh, F. Ismail, "What are Direct Medical Cost of Managing Type 2 Diabetic in Malaysia”, Information of Medical Malaysia, Vol.77, no. 5, pp.1-2, 2015

[2] T. Inoue, Y. Hatanaka, S. Okumura, C. Muramatsu, and H. Fujita, "Automated microaneurysm detection method based on eigenvalue analysis using hessian matrix in retinal fundus images," Proc. Annu. Int. Conf. IEEE Eng. Med. Biol. Soc. EMBS, pp. 5873-5876, 2013.

[3] W. A. Mustafa, H. Yazid, and S. Yaacob, "Illumination correction of retinal images using superimpose low pass and Gaussian filtering," Proc. - 2015 2nd Int. Conf. Biomed. Eng. ICoBE 2015, no. March, pp. 30-31, 2015

[4] J. Lee, S. R. Pant, and H. Lee, "An Adaptive Histogram Equalization Based Local Technique for Contrast Preserving Image Enhancement," IEEE Trans. Med. Imaging, vol. 15, no. 1, pp. 35-44, 2015.

[5] A. D. Fleming, S. Philip, K. A. Goatman, J. A. Olson, and P. F. Sharp, "Automated microaneurysm detection using local contrast normalization and local vessel detection," IEEE Trans. Med. Imaging, vol. 25, no. 9, pp. 1223-1232, 2006.

[6] M. M. Habib, R. A. Welikala, A. Hoppe, C. G. Owen, A. R. Rudnicka, and S. A. Barman, "Detection of microaneurysms in retinal images using an ensemble classifier," Informatics Med. Unlocked, vol. 9, no. May, pp. 44-57, 2017.

[7] S. S. Rahim, C. Jayne, V. Palade, and J. Shuttleworth, "Automatic detection of microaneurysms in colour fundus images for diabetic retinopathy screening," Neural Comput. Appl., vol. 27, no. 5, pp. 1149-1164, 2016.

[8] K. M. Adal, D. Sidibé, S. Ali, E. Chaum, T. P. Karnowski, and F. Mériaudeau, "Automated Detection of Microaneurysms Using Robust Blob Descriptors," Comput. Methods Programs Biomed., vol. 118, no. 1, pp. 20-45, 2015.

[9] W. Jun, and W. Shinton, "Image Thresholding using Weithed Parzen Window -Estimation". Journal of Applied Sciences, vol. 8, no. 5, pp. 772-779, 2008.

[10] Mazlan, N., Yazid, H., Rahim, S.A., Basah, S.N., "Microaneurysms segmentation in retinal images for early detection of diabetic retinopathy", Journal of Telecommunication, Electronic and Computer Engineering, 10(1-16), pp. 37-41. 2018.

[11] J. Liu, J. M. White, and R. M. Summers, "Automated detection of blob structures by hessian analysis and object scale," Proc. - Int. Conf. Image Process. ICIP, pp. 841-844, 2010.

[12] Rafael C. Gonzalez and Richard E. Woods, "Digital Image Processing", 3rd. ed. New Jersey: Pearson Practice Hall, 2008, pp. 121-123, 322-329.

[13] C. R. John, “The Image Processing Handbook,” 5rd. Ed. North Carolina: Taylor \& Francis Group, 2006 , pp. 467474.

[14] S. Jain, D. Ganotra, "Detection and Classification of Diabetic Retinopathy in Retina Image using ANN," International Journal of Scientific Reaseach in Science, Engineering, and Technology, vol. 2 ,no. 3, pp. $319-326$. 2016.

[15] K. M. Adal, D. Sidibé, S. Ali, E. Chaum, T. P. Karnowski, and F. Mériaudeau, "Automated Detection of Microaneurysms Using Robust Blob Descriptors," Comput. Methods Programs Biomed., vol. 118, no. 1, pp. 20-45, 2015. 